\title{
O MODELO CONCEITUAL FRBR: DISCUSSÕES RECENTES E UM OLHAR SOBRE AS TAREFAS DO USUÁRIO ${ }^{1}$ \\ THE CONCEPTUAL MODEL FRBR: RECENT DEBATES AND A LOOK INTO USER TASKS
}

Fernanda Passini Moreno Graduada em Biblioteconomia e Ciência da Informação pela Universidade Federal de São Carlos, Mestre em Ciência da Informação pela Universidade de Brasília Doutoranda em Ciência da Informação na mesma Universidade fpassini@gmail.com

\begin{abstract}
Resumo
Este trabalho apresenta sucintamente o modelo Requisitos Funcionais para Registros Bibliográficos (FRBR), para então apontar seus desdobramentos, isto é, os modelos que derivaram do original, alguns padrões que foram afetados após a sua publicação, trazendo algumas discussões atuais sobre estas questões. Em seguida, detalham-se as tarefas do usuário do modelo FRBR e outras idéias encontradas na literatura, bem como as tarefas do modelo Functional Requirements for Authority Data (FRAD). Concluindo, são apresentados comentários finais sobre o modelo FRBR e as tarefas do usuário.
\end{abstract}

Palavras-chave: Representação Descritiva. FRBR. FRAD. Catálogos. Tarefas do Usuário.

\section{INTRODUÇÃO}

Trabalhos anteriores já versaram sobre os conceitos e discussões que envolvem o modelo conceitual Requisitos Funcionais para Registros Bibliográficos (FRBR), dentre os quais podemos citar Moreno (2006); Silveira (2007) - que investigou os pontos de acesso de responsabilidade pessoal; e Lima (2008), que abordou a modelização dos FRBR orientado a objeto - FRBR oo $_{\text {. }}$

A produção intelectual nacional relacionada ao tema ainda é incipiente, mesmo passado tanto tempo da publicação do modelo e a despeito dos recentes esforços de algumas instituições ${ }^{2}$.

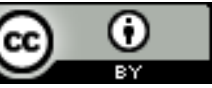
DOI 10.5007/1518-2924.2009v14n27p47

\footnotetext{
${ }^{1}$ A autora agradece à Prof. Dra. Marisa Brascher pelos comentários e sugestões que levaram à elaboração deste artigo. ${ }^{2}$ Como, por exemplo, os congressos INTEGRAR, em 2006 e CBBD - Congresso Brasileiro de Biblioteconomia, Documentação e Ciência da Informação, em 2007 - organizados pela FEBAB - Federação Brasileira de Associações de Bibliotecários, Cientistas da Informação e Instituições, que contaram com a presença de pessoas envolvidas no tema: Pat Riva e Dra. Barbara Tillett, respectivamente.

Enc. Bibli: R. Eletr. Bibliotecon. Ci. Inf., ISSN 1518-2924, Florianópolis, v. 14, n. 27, p.47-68, 2009.
} 
Considerando as pesquisas recém-concluídas mencionadas acima, não é necessário um novo detalhamento do modelo FRBR, que buscamos aqui caracterizar sucintamente, destacando os pontos de interesse para este trabalho.

Aprovado o texto final pelo Comitê Permanente em 1997, foi denominado Functional Requirements for Bibliographic Records: final report e publicado pela K. G. Saur em 1998, bem como disponibilizado on-line no site da IFLA $^{3}$, que a partir de então estabeleceu planos estratégicos para divulgação e promoção do modelo FRBR.

O modelo apresenta as 10 entidades divididas em: Grupo 1 (Obra, Expressão, Manifestação e Item), Grupo 2 (Pessoa e Entidade Coletiva), Grupo 3 (Conceito, Objeto, Evento e Lugar), além de um grupo adicional de entidades (agregadas e componentes), estas menos exploradas nos FRBR.

Como um mesmo recurso de informação pode ser desdobrado nas quatro entidades do Grupo 1, muito se questionou sobre o limite entre elas. Conforme comentado por Moreno (2006, p. 44), foram discutidos na literatura os limites e a definição de Obra - esta, por ser demasiado abstrata, pode sofrer variações de interpretação de uma cultura para outra, conforme afirmação no próprio modelo (IFLA STUDY GROUP..., 1998, p. 16) - não havendo definição operacional para esta entidade.

A entidade Expressão, em especial, foi objeto de um Grupo de Estudos de revisão, tendo Pat Riva $^{4}$ como responsável, gerando uma nova publicação do modelo atualizado (IFLA STUDY GROUP..., 2008). As inclusões mais significativas desta versão revisada dos FRBR não alteraram os limites, mas esclareceram que pequenas alterações no conteúdo intelectual da expressão são consideradas variações da mesma expressão, enquanto que no original de 1998 , qualquer mudança, por menor que pudesse parecer, seria considerada uma nova expressão na versão atual do modelo este trecho foi suprimido.

Atualmente (IFLA STUDY GROUP..., 2008, p. 20), quando uma expressão sofre acréscimos que não sejam realizações integrais de uma obra, estes são considerados para serem separados e, dependendo do grau de importância, podem ou não ter uma identificação distinta. Em outras palavras, as ilustrações contidas em expressões podem ter uma descrição à parte, desde que o bibliotecário (a) considere pertinente aos seus usuários, ou no caso do autor das ilustrações ser digno desta descrição (como Di Cavalcanti ilustrando os textos de Jorge Amado, por exemplo). O texto atual do modelo assegura a liberdade de escolha do catalogador na descrição da expressão ao não impor regras.

\footnotetext{
${ }^{3}$ International Federation of Library Associations - Site da IFLANET: $<$ http://www.ifla.org $>$.

${ }^{4}$ Atual presidente do FRBR Review Group.

Enc. Bibli: R. Eletr. Bibliotecon. Ci. Inf., ISSN 1518-2924, Florianópolis, v. 14, n. 27, p.47-68, 2009.
} 
O interesse crescente em examinar o universo bibliográfico através do olhar do usuário traduziu-se em um modelo centrado na percepção de que o registro bibliográfico deve ser útil a uma extensa gama de usuários, provendo funcionalidades para estes. Sendo assim, os Requisitos Funcionais para Registros Bibliográficos foram definidos em relação às tarefas genéricas realizadas pelos usuários, quando fazem buscas em bibliografias nacionais e catálogos de bibliotecas, ou os utilizam. As tarefas são: encontrar, identificar, selecionar e obter. O modelo apresenta um mapeamento de atributos e relacionamentos em relação às tarefas do usuário, isto é:

A estrutura provida pelo modelo serve como framework para analisar os usos que são feitos de dados bibliográficos com referência específica às entidades que são objeto de interesse do usuário, e os atributos e relacionamentos relevantes para a tarefa que está sendo desempenhada pelo usuário (IFLA, 1998, p. 82, tradução nossa).

A idéia do modelo, portanto, é uma reorganização da informação presente nos registros bibliográficos, de acordo com o entendimento que estes devem servir ao usuário final, fornecendo meios para a descoberta do universo bibliográfico onde se insere um determinado autor, suas obras e outros recursos de informação relacionados.

Quando implementado, o modelo traz alterações significativas na exibição dos resultados de uma consulta a um catálogo ou base de dados. Os resultados da consulta apresentam-se de maneira sensivelmente diferente da forma de apresentação atual, já que a adoção dos conceitos do modelo prevê uma descrição mais contida, arranjada de forma a relacionar sob uma única entrada (a obra, em geral pelo título), as diversas expressões (como as traduções), seguidas da descrição das manifestações (os suportes onde se encontram as expressões da obra) e, finalmente, indicando a localização do item. Deste modo, o usuário poderia conhecer as obras de determinado autor, por exemplo, incluindo todas as variações de língua ou formato e poderia 'navegar' por obras e expressões relacionadas. Esta proposta é um dos impactos mais notáveis do modelo, mas não o único, como se verá adiante.

Este artigo propõe apresentar as recentes discussões que envolvem o modelo e seus desdobramentos, como as derivações em desenvolvimento, as regras, princípios e formatos afetados, presentes na primeira parte deste trabalho, apresentadas na próxima seção. A segunda parte (composta pela seção 3 e subseção 3.1) traz algumas considerações sobre um dos elementos essenciais do modelo FRBR - as tarefas do usuário - através de revisão da literatura. 
Espera-se que este trabalho, ao reunir as recentes discussões e demonstrar o potencial das tarefas do usuário, em suas variadas formas apresentadas na revisão de literatura, venha a contribuir com este repensar sobre os objetivos do catálogo - e o que podemos oferecer aos usuários deste.

\section{FRBR - MODELOS DERIVADOS, PADRÕES AFETADOS E DISCUSSÕES RECENTES}

Atualmente o modelo FRBR continua sob constantes revisões e é objeto de grupos de estudos e, sobretudo, de questionamentos. Em 2002, a Seção de Catalogação da IFLA criou o Grupo de Trabalho para o futuro desenvolvimento dos FRBR. Em 2003, o grupo foi transformado em Grupo de Revisão dos FRBR e formou-se um subgrupo dedicado aos Recursos Contínuos que, atualmente realiza o estudo das entidades agregadas ${ }^{5}$ - Working Group on Aggregates. Um outro grupo foi estabelecido em 2003 para auxiliar a formulação dos FRBR orientado a objeto - FRBR $_{\text {oo. }}$ O grupo mais recente, formalizado em 2007, tem como tarefa definir namespaces apropriados para FRBR em RDF - Resource Description Framework e outras sintaxes apropriadas (IFLA CATALOGUING SECTION, 2008).

Quando da elaboração do modelo FRBR, ficou decidido que seria necessária a realização de outras pesquisas sobre registros de autoridades, bem como um maior detalhamento sobre assuntos, pouco tratados no modelo original. Encontram-se em estágios distintos de desenvolvimento dois outros modelos, ambos baseados em Entidade-Relacionamento e que, quando plenamente finalizados, comporão os fundamentos conceituais para a área de Representação e Organização da Informação.

O primeiro, denominado Functional Requirements for Authority Data (FRAD), quase concluído, está sendo desenvolvido pelo Grupo Functional Requirements and Numbering of Authority Records (FRANAR), sob os auspícios da Divisão de Controle Bibliográfico da IFLA e UBCIM, comentado neste trabalho em seção apropriada. Já o segundo, Functional Requirements for Subject Authority Records (FRSAR), pretende ser uma extensão dos modelos anteriores para classificação e indexação, contando atualmente com pouquíssima documentação relacionada, já que o Grupo de mesmo nome foi instituído em 2005. Um subgrupo criado para as User Tasks (tarefas do usuário), conduziu uma pesquisa de usuários

\footnotetext{
${ }^{5}$ São exemplos de entidades agregadas: coleções, seleções, antologias, séries, periódicos, etc (IFLA STUDY GROUP..., 2008, p. 29).

Enc. Bibli: R. Eletr. Bibliotecon. Ci. Inf., ISSN 1518-2924, Florianópolis, v. 14, n. 27, p.47-68, 2009.
} 
com intuito de mapear a realidade dos usuários, suas necessidades, etc. para refleti-las nas tarefas deste modelo, cujos resultados foram apresentados no Congresso International Society for Knowledge Organization (ISKO) e na Conferência Geral da IFLA, ambos em 2006 (IFLA FRSAR, 2007).

Nos dias atuais ${ }^{6}$, a área de Representação da Informação passa por grandes transformações: ao mesmo tempo em que estão sendo revistos os Princípios de Paris, datados de 1961, com a proposição de uma nova Declaração de Princípios, um substitutivo para o Código de Catalogação Anglo-Americano está sendo desenvolvido pelo atual Joint Steering Committee for Development of RDA - JSC ${ }^{7}$. Além disso, a versão preliminar da Descrição Bibliográfica Internacional Normalizada - ISBD consolidada, já está disponível no site da IFLA (ESCOLANO RODRÍGUEZ, 2007, IFLA STANDING COMMITTE..., 2007). Comentaremos estes temas a seguir.

A revisão dos Princípios de Paris vem sendo desenvolvida nos Encontros de Especialistas da IFLA sobre um Código de Catalogação Internacional, IFLA Meetings of Experts on an International Cataloguing Code (IME-ICC), idealizados com o intuito de criar um conjunto de novos e atualizados Princípios. Foram realizados cinco encontros, passando por diversos países $^{8}$. A declaração preliminar (IFLA CATALOGUING SECTION, 2008a) indica que:

Estes novos princípios substituem e alargam os Princípios de Paris, de apenas obras textuais a todo o tipo materiais e da simples escolha e forma de entrada até todos os aspectos dos registros bibliográficos e de autoridade utilizados em catálogos de bibliotecas. (tradução nossa)

Um dos objetivos declarados é que os Princípios serviriam como recomendações para um Código de Catalogação Internacional, já que assentados sobre o melhor da catalogação do mundo. Os princípios, ainda, baseiam-se na conveniência do usuário do catálogo e, naturalmente, incorporam os conceitos dos modelos FRBR e FRAD.

Já a ISBD, após a publicação pela IFLA na década de 70 do século passado, tornou -se uma família de padrões, no intuito de contemplar diferentes tipos de materiais e suportes. Surgiram ISBDs para Materiais Cartográficos - ISBD(CM) em 1977, para Arquivos de Computador ISBD(CF), em 1990 e outras, buscando cobrir variados recursos de informação.

Le Bœuf (2001) discutiu o impacto dos FRBR nas ISBDs, já que havia uma revisão em curso na época da elaboração e publicação do modelo. A nova versão preliminar consolidada integra, em uma única ISBD, a descrição de todos os tipos de recursos antes cobertos pelas

\footnotetext{
${ }^{6}$ Texto escrito em meados de 2008.

${ }^{7}$ Antigo Joint Steering Committee for Revision of AACR.

${ }^{8}$ Alemanha em 2003, Argentina em 2004, Egito em 2005, Coréia do Sul em 2006 e África do Sul em 2007.

Enc. Bibli: R. Eletr. Bibliotecon. Ci. Inf., ISSN 1518-2924, Florianópolis, v. 14, n. 27, p.47-68, 2009.
} 
ISBDs especializadas, ao mesmo tempo em que foram adaptados os requisitos de descrição para que estivessem em conformidade com os FRBR, segundo Escolano Rodríguez (2007), presidente do Grupo de Revisão.

Outro impacto sensível do modelo FRBR - e dos mais controversos - diz respeito ao novo código de catalogação, denominado Resource Description and Access (RDA). Esta iniciativa parece concorrer com a proposta de um Código Internacional de Catalogação mencionado anteriormente, pois vem sendo desenvolvido com o propósito de ser um código internacional, mesmo que elaborado por países anglo-saxões.

O Comitê Gestor (JSC) tem proporcionado ampla divulgação do projeto, sendo possível consultar as versões preliminares (drafts) que incluem o escopo, os objetivos, um mapeamento entre RDA e os FRBR, entre outros. A previsão é que o novo código esteja concluído em 2009 e inclua uma ferramenta eletrônica para sua consulta, baseada em XML EXtensible Markup Language. Segundo os comentários de diversos membros da lista de discussão sobre $\mathrm{RDA}^{9}$, ainda não há consenso sobre se haverá versão impressa do novo conjunto de regras, nem sobre os custos de aquisição individuais ou para bibliotecas de menor porte.

Segundo Tillett (2007, p. 91), o termo resource (recurso) foi adotado para nomear o novo código por expressar melhor os materiais presentes em coleções de bibliotecas ou "as coisas que venham a ser parte do grande universo bibliográfico". Afirma ainda que "outra recomendação foi usar somente os termos dos FRBR quando eles refletissem corretamente a intenção da regra". Na obra supracitada, encontramos Yee (2007, p. 125), discutindo sobre imagens em movimento e as questões relacionadas de conteúdo e suporte no RDA:

As entidades dos FRBR foram escassamente referenciadas no texto, e o status quo foi mantido; isto é, qualquer mudança na manifestação (suporte) resulta em criação de um novo registro bibliográfico. Isso é um desastre para os usuários de catálogos interessados em obras prolíficas que existem em múltiplas expressões e manifestações (e estas são as mais populares entre os usuários) ${ }^{10}$. É também um desastre para qualquer arquivo de preservação audiovisual que faça múltiplas cópias em múltiplos formatos físicos no curso do processo de preservação. (tradução nossa)

Recentemente, o Grupo de Estudos sobre o Futuro do Controle Bibliográfico ${ }^{11}$ da Library of Congress (LC), entre muitas outras críticas e sugestões, recomendou a suspensão dos

\footnotetext{
${ }^{9}$ Disponível em: $<$ http://www.collectionscanada.gc.ca/jsc/rdadiscuss.html $>$.

${ }^{10}$ Já Žumer (2007, p. 28-), ao levantar os dados da pesquisa de Bennett, Lavoie, O’Neill (2003), afirma que a maior parte das obras tem apenas uma expressão e manifestação correspondentes - no catálogo da OCLC, WorldCat apenas $20 \%$ de todas as obras têm mais de uma manifestação e $1 \%$ mais de sete.

11 Library of Congress Working Group on the Future of Bibliographic Control, que atende pela excêntrica sigla WoGroFuBiCo. O Grupo incluiu, além de bibliotecários de várias instituições e universidades norte-americanas, representantes de empresas da área de tecnologia (Google e Microsoft).

Enc. Bibli: R. Eletr. Bibliotecon. Ci. Inf., ISSN 1518-2924, Florianópolis, v. 14, n. 27, p.47-68, 2009.
} 
trabalhos com o RDA, basicamente porque este se baseia em um modelo pouco testado e implementado, qual seja, o modelo FRBR (IFLA WORKING GROUP...., 2008, p. 29, 33). A LC constituiu um grupo interno para analisar o relatório daquele grupo de estudos, e as respostas aos questionamentos e sugestões foram redigidas e compiladas por Marcum (2008, p. 51).

Segundo as respostas dadas por Marcum, o desenvolvimento do RDA não seria suspenso, dado o avançado estágio de desenvolvimento, mas o grupo da LC, reconhecendo a insuficiência de testes com os FRBR (MARCUM, op.cit.), assumiu o compromisso de dedicar-se à questão de possíveis implementações e algoritmos de FRBR-ização.

Esta crítica ao modelo FRBR é compreensível: passados exatos 10 anos de sua publicação, praticamente um desenvolvedor de software tem condições de dizer que seu produto é totalmente baseado nos FRBR - o responsável pela empresa foi membro do grupo de estudos para o desenvolvimento do modelo. Há diversos projetos de estudos de implementação, mas iniciativas como RedLightGreen, que possibilitava a busca em múltiplos catálogos em uma única interface baseada nos FRBR, agora integra os projetos da Online Computer Library Center (OCLC), e parece estar ainda em fase de transição. O Morbus Iff's LibDB project ou LibDB, projeto independente paralisado a algum tempo, voltou à ativa em março de 2007 e o Open FRBR, projeto em andamento de William Denton, responsável pelo FRBR blog (assim como outros não mencionados aqui) são iniciativas quase individuais, que carecem de recursos ou infra-estrutura para um completo desenvolvimento.

Žumer (2007, p. 27), aponta que "passados quase dez anos, nós ainda nos referimos freqüentemente aos FRBR como 'o novo modelo de biblioteca'. Isso pode ser explicado pelo fato de não haver muitas implementações reais dos FRBR.” Reforçando este argumento, um estudo Delphi realizado pela equipe da Kent State University (ZHANG; SALABA, 2007), com intuito de verificar questões críticas sobre os FRBR, demonstrou a preocupação dos especialistas consultados: o desenvolvimento de sistemas baseados no modelo está presente em quatro dos dez principais itens apontados por estes.

Entre os algoritmos já disponíveis, o Work-set da Online Computer Library Center (OCLC) é um exemplo que atua no nível da Obra e não se pode afirmar que seja uma FRBR-ização de fato $^{12}$ (WORKING GROUP...., 2008, p. 33). A ferramenta conversora utilizada por Moreno (2006), FRBR Display Tool, não possibilita a conversão completa de um catálogo, não implica em modificações no trabalho de catalogação e nem se pretende um software para

\footnotetext{
${ }^{12}$ Assunto também comentado nos blogs FRBR $(<\mathrm{http}: / /$ www.frbr.org $>)$ e Bibliographic Wilderness $(<\mathrm{http}: / /$ bibwild.wordpress.com/>).

Enc. Bibli: R. Eletr. Bibliotecon. Ci. Inf., ISSN 1518-2924, Florianópolis, v. 14, n. 27, p.47-68, 2009.
} 
gestão de registros bibliográficos - apenas exibe os registros inseridos de forma modelada 'à la FRBR.’

Não parece ser a intenção do Grupo de Estudos sobre o Futuro do Controle Bibliográfico, e certamente não é a nossa, uma desqualificação do modelo - apenas concordamos que os FRBR continuam sendo excelente modelo conceitual.

Modelos podem ser entendidos como uma forma de compreender a realidade. Para McQuail e Windahl (apud Le Coadic, 1996, p. 72) "um modelo permite interpretar um conjunto de fenômenos por meio de uma estrutura da qual mostra os principais elementos e as relações existentes entre tais elementos", qual seja, um esquema que pode possibilitar a representação de um fenômeno. O uso de modelos na ciência tem abordagens distintas e não é consensual entre pesquisadores. Estudiosos de várias áreas do conhecimento valem-se de modelos por entenderem que:

Os modelos são aproximações altamente subjetivas, no sentido de não incluírem todas as observações, mensurações e medições associadas, mas, como tais, são valiosos por ocultarem detalhes secundários e permitirem o aparecimento dos aspectos fundamentais da realidade. (CHORLEY; HAGGETT, 1975, p. 4)

Segundo Carlyle (2006, p. 265), “em essência, o FRBR é um modelo de um modelo, se considerarmos que um registro bibliográfico é uma representação de um documento, e da sua própria maneira, é mais um modelo como FRBR". O autor segue afirmando que modelos podem predizer um comportamento, eventos ou outros fenômenos e ainda melhorar produtos, processos e tecnologias, concluindo que o "FRBR é um modelo conceitual cujo principal propósito é melhorar registros de catalogação (um produto), a catalogação (um processo), e os catálogos (uma tecnologia)". O autor afirma que o que é novo e diferente no modelo FRBR é que este identifica e define explicitamente quatro entidades, as quais reconhece simultaneamente, e apresenta um modelo de catalogação baseado no modelo EntidadeRelacionamento.

Acreditamos que, a despeito das críticas, deve-se reconhecer o impacto do modelo na área de organização e representação da informação. A partir das idéias oriundas de uma área constantemente desprivilegiada e praticamente invisível, como a representação descritiva, o modelo despertou a comunidade profissional e acadêmica para um tema comum: a organização da informação, ao retomar princípios e proposições consagradas, revestindo-os de uma nova linguagem e ampliando seu objetivo e alcance. Entre outros pontos que poderiam ser aqui elencados, destacamos que o modelo FRBR trouxe inúmeras mudanças, promovendo:

Enc. Bibli: R. Eletr. Bibliotecon. Ci. Inf., ISSN 1518-2924, Florianópolis, v. 14, n. 27, p.47-68, 2009. 
- um melhor entendimento dos relacionamentos entre os recursos de informação registrados, privilegiando o conteúdo, isto é, por repensar o objeto bibliográfico (agora recurso de informação), dotado de quatro dimensões, relacionado com o universo que o cerca;

- um olhar diferenciado sobre os diversos responsáveis pelo recurso de informação, aferindo o mesmo status a intérpretes, autores, tradutores, executores, etc.;

- o desenvolvimento de outros modelos a partir do know-how adquirido, como extensões do FRBR, ao modelar registros de autoridade (FRAD) e assuntos (FRSAR), fornecendo fundamentos conceituais para área;

- um movimento intenso de saudáveis discussões na área de organização e representação, levando à revisão de regras, padrões e modelos (AACR/RDA, ISBDs variadas/ISBD consolidada, MARC);

- uma aproximação com diversas comunidades: na área de tecnologia, por ser baseado em um assentado modelo computacional, e na área de museus com a harmonização para o FRBR;

- e, finalmente, podemos afirmar que o modelo proporcionou uma tomada de consciência de um fato que é constantemente difundido e pouco praticado, qual seja, de que as diversas formas de organização da informação devem servir ao usuário.

\section{AS TAREFAS DOS USUÁRIOS - USER TASKS}

Curiosamente, os conceitos menos explorados na literatura sobre os FRBR são justamente as tarefas do usuário: a bibliografia referente ao modelo - um exercício de controle bibliográfico empreendido pela IFLA (IFLA CATALOGUING..., 2007) - não traz uma seção dedicada, levando o interessado no assunto a vasculhar incessantemente outros artigos, ou deparar-se com o tema por acaso. É preciso notar que esta bibliografia compilada é vasta, oferecendo uma grande cobertura de assuntos, como aspectos teóricos, ensino, páginas web e blogs dedicados aos FRBR, relações com outros modelos e tópicos, entre outros.

Nesta seção buscamos trazer uma revisão da literatura recente sobre as tarefas do usuário presentes no modelo FRBR e analisamos as tarefas presentes no modelo ampliado de Delsey (2003), como extrato do levantamento de pesquisa em andamento. A subseção 3.1 apresenta 
as tarefas do usuário no modelo ainda em desenvolvimento, Functional Requirements for Authority Data (FRAD), onde se pontua a ausência de discussões acerca do tema.

Como mostrado anteriormente, os FRBR foram definidos em relação às tarefas genéricas realizadas pelos usuários, chamadas user tasks, no intuito de atender suas necessidades informacionais. Espera-se que os usuários façam uso dos dados contidos em um registro para encontrar, identificar, selecionar e obter os recursos desejados. De forma mais detalhada, segue a descrição contida no Relatório Final do modelo FRBR.

\begin{tabular}{c|l}
\hline Encontrar & $\begin{array}{l}\text { Encontrar uma única entidade ou um conjunto de entidades em um arquivo ou base de dados } \\
\text { como o resultado de uma busca usando um atributo ou o relacionamento da entidade; }\end{array}$ \\
\hline Identificar & $\begin{array}{l}\text { Confirmar que a entidade descrita corresponde à entidade procurada, ou para distinguir entre } \\
\text { duas ou mais entidades com características similares. }\end{array}$ \\
\hline Selecionar & $\begin{array}{l}\text { Selecionar uma entidade adequada às necessidades do usuário, isto é, para escolher uma entidade } \\
\text { que vá ao encontro das exigências do usuário em relação ao conteúdo, formato físico, etc., ou à } \\
\text { rejeição de uma entidade como sendo imprópria às necessidades do usuário; }\end{array}$ \\
\hline Obter & $\begin{array}{l}\text { Encomendar, adquirir, ou obter acesso à entidade descrita, isto é, para adquirir uma entidade } \\
\text { através de compra ou empréstimo, etc., ou para acessar eletronicamente uma entidade através de } \\
\text { uma conexão em linha a um computador remoto. }\end{array}$ \\
\hline
\end{tabular}

Tabela 1: Tarefas do usuário FRBR

Fonte: IFLA (1998, p. 8; 82). Tradução nossa.

Nota-se que as tarefas genéricas acima descritas são fortemente inspiradas nos três objetivos ${ }^{13}$ do catálogo, propostos por Cutter. Segundo Denton (2007, p. 40) estas são algumas das palavras mais citadas da história da biblioteconomia, quais sejam:

1) Permitir a uma pessoa encontrar um livro do qual:
(A) o autor
(B) o título
(C) assunto
\} seja conhecido

2) Mostrar o que a biblioteca possui:

(D) de um autor determinado;

(E) um assunto determinado;

(F) em um tipo dado da literatura;

3) Para ajudar na escolha de um livro:

(G) de acordo com sua edição (bibliograficamente);

(H) de acordo com seu caráter (literário ou tópico).

De acordo com Svenonius (2000, p. 15-16), o primeiro objetivo pode ser entendido como o objetivo de encontrar (finding objective), supondo que o usuário tem em mãos um autor, título ou assunto e está procurando um documento. O segundo seria o objetivo de organizar (collocating objective), onde se assume que o usuário tem em mãos informações semelhantes, mas necessita de um conjunto de documentos, seja de um dado autor, assunto ou gênero. $\mathrm{O}$

\footnotetext{
${ }^{13}$ Mey (1987, p.18) nota que: “A tradução literal seria “objetos”. A palavra apresenta os dois sentidos (assunto, tema, objeto) e Cutter utiliza ambos os sentidos no texto. Mas preferiu-se traduzi-la dessa forma [objetivos], mais representativa da idéia aqui exposta".

Enc. Bibli: R. Eletr. Bibliotecon. Ci. Inf., ISSN 1518-2924, Florianópolis, v. 14, n. 27, p.47-68, 2009.
} 
terceiro seria o objetivo da escolha (choice objective), no qual supostamente o usuário estaria diante de um número similar de documentos e necessitaria fazer uma escolha efetiva entre eles como, por exemplo, entre diversas edições de uma obra.

Para Denton (2007, p. 42), o conjunto de tarefas do modelo FRBR é mais amplo que as propostas feitas por Cutter, e dão ao usuário muito mais liberdade, permitindo a busca por qualquer atributo da entidade. Em seguida (idem, p. 44), comenta que uma boa implementação das tarefas pouparia o tempo do usuário - a quarta das cinco leis de Ranganathan.

Nos Princípios de Paris, que abrangem apenas os elementos principais que determinam a ordem da entrada nos catálogos de apenas um tipo de material (livros impressos); também vemos que o catálogo deverá ser um instrumento eficaz para determinar:

2.1 se a biblioteca possui um determinado livro especificado por:

a) seu autor ou título, ou

b) - se o autor ou o título são inapropriados ou insuficientes para sua

identificação - um substituto conveniente;

2.2 e ainda,

a) que obras existem de um determinado autor e;

b) que edições de uma determinada obra existem na biblioteca (DECLARACIÓN...., p.1, 2007, tradução livre).

Segundo Svenonius (2000, p. 15-16), os objetivos declarados nos Princípios de Paris modificaram ligeiramente a proposta de Dewey, pois foram baseados na proposta de Lubeztky. Denton (2007, p. 47) nota que a substituição da palavra 'livro' por 'obra' foi o 'conserto' na 'falha' que Lubeztky via na proposta de Cutter. Voltando à Svenonius (idem), esta ressalta que os objetivos de Cutter foram respeitados por 75 anos, até a alteração proposta nos Princípios acima citados, que só sofreram nova modificação com a proposta das tarefas do usuário do modelo FRBR. Estas tarefas têm sofrido sugestões e propostas de ampliação e a literatura aponta outras idéias, comentadas nos parágrafos a seguir.

$\mathrm{Na}$ análise funcional desenvolvida por Delsey (2003) que mapeou o formato MARC em relação aos FRBR, constava o objetivo de "desenvolver um modelo (baseado nos FRBR) que refletisse toda a extensão dos dados contidos nos formatos do MARC" (DELSEY, 2003, p. 5; MORENO, 2006, p. 79).

Nesta ampliação do modelo, o autor propôs outros diagramas; criou entidades associadas à Obra; estabeleceu novos relacionamentos e forneceu definições para cada um deles. Tomamos como exemplo a entidade 'Obra': é uma entidade FRBR, usada em (relacionamento Delsey) um programa de estudos (entidade Delsey) que é parte de (relacionamento Delsey) um currículo (entidade Delsey).

Enc. Bibli: R. Eletr. Bibliotecon. Ci. Inf., ISSN 1518-2924, Florianópolis, v. 14, n. 27, p.47-68, 2009. 
Ao mapear os elementos de dados MARC às tarefas dos usuários, o autor fez uma correlação entre faixas de campos (por exemplo, campos de descrição física 300-362) em relação às doze tarefas, que refletem o processamento e o uso dos recursos. O autor apresenta doze tarefas do usuário, onde três são idênticas às do modelo FRBR (e uma modificada, comentada adiante) e as demais são novas tarefas, agrupadas em três categorias: descoberta de recurso, uso do recurso e administração de dados. Comentamos a primeira categoria após a tabela abaixo.

\begin{tabular}{c|l}
\hline Pesquisar & $\begin{array}{l}\text { Pesquisar por um recurso que corresponda a um dado critério (i.e, pesquisar tanto por uma } \\
\text { simples entidade ou por um conjunto de entidades usando um atributo ou relacionamento da } \\
\text { entidade como critério de busca) }\end{array}$ \\
\hline Identificar & $\begin{array}{l}\text { Identificar um recurso (i. e., confirmar que aquela entidade descrita ou localizada corresponde à } \\
\text { entidade buscada, ou distinguir entre duas ou mais entidades com características similares). }\end{array}$ \\
\hline Selecionar & $\begin{array}{l}\text { Selecionar um recurso que seja apropriado às necessidades do usuário (i.e., escolher uma } \\
\text { entidade que vá ao encontro dos requisitos do usuário com respeito ao conteúdo, formato físico, } \\
\text { etc., ou rejeitar a entidade como sendo inapropriada às necessidades do usuário). }\end{array}$ \\
\hline Obter & $\begin{array}{l}\text { Acessar um recurso tanto física quanto eletronicamente através de uma conexão online a um } \\
\text { computador remoto, e/ou adquirir um recurso através de aquisição, licença, empréstimo, etc. }\end{array}$ \\
\hline Tabela 2: Tarefas do usuário - categoria descoberta de recurso \\
Fonte: Delsey (2003, p. 10). Tradução nossa.
\end{tabular}

De forma simplificada Delsey retoma as quatro tarefas dos FRBR, não alterando substancialmente as tarefas identificar, selecionar e obter. O destaque fica para a tarefa 'encontrar' modificada para 'pesquisar'. Ao pensarmos neste conjunto de tarefas como ações do usuário, de fato deve ser feita a pesquisa na base de dados, catálogo ou outro sistema, para que este possa retornar o "recurso que corresponda a dado critério" ou u ma lista de referências de recursos. Nesse sentido, a tarefa 'encontrar' como originalmente expressa no modelo FRBR, seria o passo seguinte ou uma sub-tarefa da atividade de pesquisar, que podem representar a primeira etapa de interação usuário-sistema.

Ao agrupá-las sob o título 'descoberta de recursos de informação', acreditamos que o autor proporciona um melhor entendimento da função primordial das tarefas, atendendo aos objetivos do catálogo como propostos por Cutter, conforme comentado anteriormente. A segunda categoria é apresentada a seguir.

\begin{tabular}{l|l}
\hline Restringir & $\begin{array}{l}\text { Controlar o acesso ou uso de um recurso (isto é, restringir acesso e/ou uso de uma entidade } \\
\text { com base em direitos de propriedade, política administrativa, etc.) }\end{array}$ \\
\hline Administrar & Administrar um recurso em curso de aquisição, circulação, preservação, etc. \\
\hline Operar & $\begin{array}{l}\text { Operar um recurso (isto é, abrir, exibir, tocar }{ }^{14} \text {, ativar, fazer funcionar }{ }^{15} \text {, uma entidade que } \\
\text { requeira equipamento especializado, software, etc. para esta operação) }\end{array}$ \\
\hline Interpretar & Interpretar ou avaliar a informação contida em um recurso \\
\hline Tabela 3: Tarefas do usuário - categoria uso do recurso \\
\hline${ }^{14}$ Play, no original. \\
${ }^{15}$ Run, no original. \\
Enc. Bibli: R. Eletr. Bibliotecon. Ci. Inf., ISSN 1518-2924, Florianópolis, v. 14, n. 27, p.47-68, 2009.
\end{tabular}


O grupo de tarefas que são destinadas ao uso dos recursos de informação engloba aquelas desempenhadas pelos profissionais envolvidos no tratamento do recurso, isto é, o trabalho técnico e administrativo que permite: restringir o uso por terceiros, administrar o recurso durante o workflow do processamento técnico e operar um recurso que necessite ser executado.

A última tarefa - interpretar - é a mais problemática nesta esquematização, já que seu objetivo não foi detalhado. Em um primeiro momento, parece envolver diversas etapas do trabalho intelectual que pode ser desempenhado por mais de um profissional, se compreendermos esta tarefa como as atividades de indexação, classificação e catalogação.

Sendo isso uma inferência, consultamos o mapeamento destas tarefas em relação aos campos MARC (DELSEY, 2003, p. 13) na tentativa de descobrir o objetivo de 'interpretar'. O autor selecionou os campos 250-270 (edição, imprenta, etc.) e a faixa de campos de notas (500-586) como de uso secundário para estas tarefas, não sendo indicados os de uso principal ou mais freqüente, o que contraria nossa idéia inicial, pois não está relacionada a todos os campos de descrição utilizados pelo catalogador, por exemplo. Não foram encontrados apontamentos sobre estas idéias na literatura, prejudicando a possibilidade de confrontar nosso pensamento com o de outros pesquisadores.

Consideramos esta proposta válida, mas é preciso notar que o termo 'uso' pode nos remeter, a princípio, ao uso que usuários reais ou potenciais podem vir a fazer daquele recurso selecionado ou obtido. Dado o caráter das tarefas, "administração dos recursos" nos parece uma expressão mais apropriada para categorizá-las. Não obstante, o autor propõe também tarefas relacionadas à administração de dados, apresentadas e comentadas a seguir.

\begin{tabular}{c|l}
\hline Identificar & $\begin{array}{l}\text { Identificar um registro, segmento, campo, ou elemento de dado (isto é, diferenciar um } \\
\text { componente lógico de dados de outro) }\end{array}$ \\
\hline Processar & $\begin{array}{l}\text { Processar um registro, segmento, campo, ou elemento de dado (isto é, adicionar, apagar, } \\
\text { substituir, emitir }{ }^{16} \text {, etc. um componente lógico de dados através de um processo automatizado) }\end{array}$ \\
\hline Ordenar & Classificar um campo com a finalidade de uma organização alfabética ou numérica \\
\hline Exibir & $\begin{array}{l}\text { Exibir um campo ou um elemento de dado (isto é, exibir um campo ou elemento de dado com } \\
\text { uma constante de impressão adequada ou como um traçado/desenho) }\end{array}$ \\
\hline
\end{tabular}

O terceiro conjunto de tarefas procurou identificar atividades relacionadas ao tratamento de dados. Para a tarefa identificar, foram relacionadas as faixas de campos MARC de controle

\footnotetext{
${ }^{16}$ Output, no original.

Enc. Bibli: R. Eletr. Bibliotecon. Ci. Inf., ISSN 1518-2924, Florianópolis, v. 14, n. 27, p.47-68, 2009.
} 
(001-005) e, números e códigos (010-088), indicados como de uso principal e secundário, respectivamente. A tarefa processar é relacionada ao Líder, além destas acima mencionadas. As demais tarefas não foram mapeadas.

Conforme afirmamos em outras oportunidades neste trabalho, a literatura sobre as tarefas do modelo FRBR é relativamente escassa e não encontramos discussões acerca das oito novas propostas feitas por Delsey.

No entanto, há outros autores que reconhecem a insuficiência das quatro tarefas iniciais do modelo FRBR: Svenonius (2000) manifestou a idéia de uma quinta tarefa na obra "The intellectual foundation of information organization." A autora advoga pela inclusão da tarefa 'navegar', constituindo uma argumentação dividida em duas partes: a primeira é delineada pelas pesquisas sobre comportamento de busca dos usuários; a segunda analisa os códigos tradicionais de descrição bibliográfica. A autora faz um paralelo entre o caminhar pelas estantes em uma biblioteca - "o microcosmo do universo bibliográfico" (idem, p. 19) - onde o usuário é 'guiado' pela classificação, que organiza e relaciona os livros por assunto. A segunda parte do argumento consiste no entendimento que:

Os códigos de regras bibliográficas "costumam organizar os documentos supondo sua existência. (...) Eles incluem regras que especificam relacionamentos entre obras bem como relacionamentos entre nomes de atributos de obras, como autores e assuntos. (...) O objetivo das regras determinarem esses relacionamentos é mapear o universo bibliográfico - isto é, facilitar a navegação.” (idem, p. 19)

Svenonius (2000, p. 20) propõe, então, uma definição para a nova tarefa: "Navegar uma base de dados bibliográfica (isto é, achar obras relacionadas a uma dada obra por generalização, associação ou agregação; encontrar atributos relacionados por equivalência, associação ou hierarquia)."

A idéia foi retomada em 2005, durante o encontro do FRBR Review Group reunidos em Olso, quando foram analisadas e discutidas algumas propostas em relação às tarefas do usuário (IFLA CATALOGUING..., 2005). Apresentamos as discussões resumidamente nos tópicos abaixo:

- Adicionar a tarefa navegar: Em resumo, foi argumentado que esta tarefa estava "implicitamente contida nos relacionamentos", que "navegar em um catálogo" não era um objetivo em si mesmo e que o ambiente Web talvez tenha mudado os comportamentos e expectativas sobre a forma como a informação é apresentada. A decisão do grupo foi que Barbara Tillett e Patrick Le Bœuf proporiam uma forma de 
clarear o argumento de que esta é uma tarefa implícita em uma mais ampla encontrar - negando sua inclusão, portanto.

- Inclusão das tarefas "atribuir royalties a" e "preservar": O Grupo concordou que estas tarefas são relevantes, mas aplicam-se a determinados contextos, negando sua inclusão.

Uma terceira proposta foi feita pela então presidente do Grupo de Estudos para Ensino dos FRBR, Maja Žumer ${ }^{17}$. O grupo decidiu que o tema deveria ser desenvolvido futuramente:

Do ponto de vista metodológico, o modelo FRBR foi desenvolvido com base nos documentos normativos existentes, identificando os elementos de dados que satisfaçam cada tarefa do usuário para cada entidade; não seria melhor tomar as tarefas do usuário como base, e listar todos os elementos que melhor se ajustariam àquelas tarefas? Desse modo, poderíamos assegurar-nos que os elementos de dados não se perderiam nos documentos normativos atuais. (IFLA CATALOGUING..., 2005, p. 6)

Durante a discussão, Maja acrescentou que muitos usuários consultam a Amazon ao invés de catálogos de bibliotecas, talvez porque o último não inclua todos os elementos de informação importantes. Infelizmente a discussão não foi retomada, já que nos dois encontros seguintes (IFLA CATALOGUING..., 2007), realizados respectivamente em Seul e Durban, não foram abordados assuntos diretamente relacionados às tarefas.

Entretanto, parece que a comunidade dedicada à catalogação reconheceu que navegar é parte do comportamento de busca de usuário, seja pela experiência no uso da Web, seja 'navegando' nas estantes como pontuado por Svenonius: o futuro Código de Catalogação Internacional, mencionado anteriormente neste trabalho, propõe a agregação da tarefa 'navegar' às quatro já existentes nos FRBR, na última versão da declaração disponível (IFLA STATEMENT...., 2008).

Note-se ainda, que no futuro Código de Catalogação Internacional, a tarefa 'identificar' foi dividida em "localizar um único recurso" e "localizar um conjunto de recursos", baseados nas propostas de Svenonius (2000), comentada neste trabalho: finding objective e collocating objective.

O modelo Functional Requirements for Authority Data (FRAD) também apresenta tarefas do usuário. $\mathrm{Na}$ próxima seção, intitulada "Outro modelo, outras tarefas" apresentamos sinoticamente o modelo, traçando um recorte nos conceitos apropriados a este estudo. Por não haver uma versão final deste modelo, baseamos nossa explanação no último draft disponível, de abril de 2007.

\footnotetext{
${ }^{17}$ Atualmente é presidente do Grupo de Trabalho "Guidelines for national bibliographies in the digital age”, vice-presidente do Grupo de Trabalho FRSAR e colaboradora no desenvolvimento do FRBR ${ }_{\mathrm{oo}}$.

Enc. Bibli: R. Eletr. Bibliotecon. Ci. Inf., ISSN 1518-2924, Florianópolis, v. 14, n. 27, p.47-68, 2009.
} 


\subsection{Outro modelo, outras tarefas: Functional Requirements for Authority Data (FRAD)}

O Termo de Referência para o Grupo de Trabalho Functional Requirements and Numbering of Authority Records (FRANAR), constituído em 1999, declara que a função do modelo FRAD é "definir requisitos funcionais para registros de autoridade, continuando o trabalho iniciado pelos FRBR" (LE BCEUF, 2007). As entidades do Grupo 2 dos FRBR (Pessoa e Entidade Coletiva), representadas por cabeçalhos, foram completamente modeladas, agregando-se a entidade "Família", em diagramas Entidade-Relacionamento.

Em 2003, o modelo era denominado FRANAR, como o nome do grupo de estudos (PATTON, 2003). Em 2005, a primeira versão preliminar do modelo, então chamado Functional Requirements for Authority Records (FRAR) foi disponibilizado para revisão e a segunda versão, agora novamente renomeado (FRAD), encontra-se neste processo desde abril de 2007.

O grupo também desenvolveu tarefas do usuário, à semelhança das encontradas nos FRBR (IFLA FRANAR, 2007, p. 50) e definiu qual o grupo de usuários a que se destinam os dados de autoridade.

\footnotetext{
Para os propósitos desta análise, os usuários de dados de autoridade são amplamente definidos a fim de incluir:

- criadores de dados de autoridade que criam e mantém arquivos de autoridade;

- usuários que utilizam informação de autoridade através de acesso direto aos arquivos de autoridades ou indiretamente através do ponto de acesso controlado (formas autorizadas, referências, etc.) em catálogos, bibliografias nacionais, outras bases de dados similares, etc.(IFLA FRANAR, 2007, p. 50).
}

Segundo Patton (2003, p. 46), no FRAD, como no modelo FRBR, as entidades, atributos e relacionamentos foram mapeados em relação às tarefas do usuário, com a intenção de explicar como os dados de autoridade apóiam cada tarefa do usuário específica. De acordo com o foco deste estudo, são apresentadas as quatro tarefas, sistematizadas na tabela abaixo.

\begin{tabular}{c|l}
\hline Encontrar & $\begin{array}{l}\text { Encontrar uma entidade ou conjunto de entidades correspondentes ao critério dado (isto é, } \\
\text { encontrar uma única entidade ou um conjunto de entidades usando um atributo ou uma } \\
\text { combinação de atributos ou relacionamentos da entidade como critério de busca); ou } \\
\text { explorar o universo de entidades bibliográficas usando aqueles atributos e relacionamentos. }\end{array}$ \\
\hline Identificar & $\begin{array}{l}\text { Identificar uma entidade (isto é, confirmar que aquela entidade representada corresponde à } \\
\text { entidade procurada, distinguindo entre duas ou mais entidades com características similares) } \\
\text { ou validar a forma do nome a ser usada como ponto de acesso controlado. }\end{array}$ \\
\hline Contextualizar & $\begin{array}{l}\text { Situar uma pessoa, entidade coletiva, obra, etc. em um contexto; evidenciar o } \\
\text { relacionamento entre uma ou mais pessoas, entidades coletivas, obras, etc. e um nome pelo }\end{array}$ \\
\hline
\end{tabular}

Enc. Bibli: R. Eletr. Bibliotecon. Ci. Inf., ISSN 1518-2924, Florianópolis, v. 14, n. 27, p.47-68, 2009. 


\begin{tabular}{cl}
\hline & $\begin{array}{l}\text { qual aquela pessoa, entidade coletiva, etc. é conhecida (por exemplo, nome usado na } \\
\text { religião versus nome secular) }\end{array}$ \\
\hline Justificar & $\begin{array}{l}\text { Documentar a razão da escolha, pelo criador do dado de autoridade, de um nome ou uma } \\
\text { forma do nome pelo qual o ponto de acesso controlado foi baseado. }\end{array}$ \\
\hline Tabela 5: Tarefas do usuário FRAD \\
Fonte: IFLA FRANAR, 2007, p. 50.
\end{tabular}

As primeiras duas tarefas não diferem tanto da proposta dos FRBR, havendo no caso da tarefa identificar apenas um acréscimo sobre a validação da forma do nome, donde se conclui que a tarefa identificar são válidas para ambos os usuários descritos anteriormente: o criador do registro e o usuário. As duas últimas tarefas referem-se exclusivamente ao trabalho do criador do registro, isto é, criar relacionamentos ao contextualizar o nome adotado e, em seguida, justificar a escolha daquele nome.

A literatura aponta quase nenhuma discussão e, como o modelo não está concluído, nos abstemos de investigar o mapeamento entre a tríade entidades-atributos-relacionamentos e as tarefas. Encontramos apenas as sugestões de representantes da Biblioteca Nacional da Alemanha: como comentário geral, notam a ausência de outras tarefas adotadas na futura Declaração de Princípios Internacionais de Catalogação, oriundas dos FRBR e sugerem desdobrar a primeira tarefa em duas, isto é, que "explorar o universo de entidades bibliográficas usando aqueles atributos e relacionamentos" seja uma outra tarefa (HENGEL; HENZE; PFEIFER, 2007).Conforme apontado, o nome do novo modelo já foi modificado algumas vezes, porém as tarefas permanecem idênticas ao relato publicado em 2003.

\section{COMENTÁRIOS FINAIS}

O modelo FRBR permanece sob revisões e atualizações, incluindo questionamentos sobre o mesmo e sobre o entorno afeto a ele, como demonstramos na primeira parte deste trabalho. As fontes aqui reunidas demonstraram as controvérsias geradas na sua adoção como estrutura para o novo código de catalogação, bem como a amplitude do impacto do modelo na área de representação.

As tarefas do usuário, objeto da segunda parte deste trabalho, apesar de pouco destacadas na literatura referente aos FRBR e suas extensões, nos parecem de grande importância ao retomarem os princípios básicos da organização da informação e, principalmente, por traduzirem os objetivos do catálogo: servir ao usuário. 
É preciso lembrar que as tarefas dos usuários propostas no modelo FRBR são tarefas genéricas, isto é, independente da organização da informação em que se baseia um catálogo, acredita-se que estas tarefas básicas podem ou devem ser desempenhadas pelo usuário na descoberta de recursos de informação. Dito de outra forma, os sistemas de informação - um catálogo, uma biblioteca digital, as bibliografias nacionais, etc. - devem permitir ao usuário a descoberta de informação ao encontrar e identificar um recurso, proporcionar a chance de escolher se o recurso lhe é apropriado ou não e, finalmente, que informe como pode obtê-lo seja por um número de chamada que o leve à estante correta, seja através de um link com acesso ao texto completo do recurso buscado.

Em uma época em que o usuário de serviços de informação dispõe de tantas opções e possibilidades de busca - que incluem a visualização da capa e conteúdo básico do livro através de lojas virtuais, podendo folheá-lo como se caminhasse entre estantes - faz-se necessário repensar o papel dos catálogos no dias atuais. A proposta dos FRBR já afirmava isso dez anos atrás, e ainda assim, discute-se esta questão urgente em uma das bibliotecas que tem o maior acervo do planeta - a Library of Congress. O acréscimo da tarefa navegar, originalmente proposta por Svenonius (op. cit.) e adotada na Declaração Internacional de Princípios de Catalogação parece ir ao encontro deste cenário.

Finalizando, esperamos que este trabalho, ao reunir as recentes discussões e demonstrar o potencial das tarefas do usuário, em suas variadas formas apresentadas na revisão de literatura, venha a contribuir com este repensar sobre os objetivos do catálogo - e o que podemos oferecer aos usuários deste.

\section{REFERÊNCIAS}

BENNETT, R., LAVOIE, B., O'NEILL, E.. The concept of work in WorldCat. Library Collections, Acquisitions and Technical Services, v. 2, n.1, p. 49-59, 2003.

CARLYLE, A. Understanding FRBR as a conceptual model: FRBR and the Bibliographic Universe. Library Resources \& Technical Services, v. 50 n. 4, out. 2006, p. 264-273.

CHORLEY, R.; HAGGETT, P. Modelos, paradigmas e a nova geografia. In: CHORLEY, Richard, HAGGETT, Peter. Modelos sócio-econômicos em geografia. Rio de Janeiro: Livros Técnicos e Científicos/USP, 1975. 
CUTTER, C. A. Rules for a dictionary catalog. 4a. ed., rewritten, Washington D.C.: Government Printing office, 1904.

DECLARACIÓN de Principios Adoptados por la Conferencia Internacional sobre Principios de Catalogación Paris, 1961. Traducción: Elena Escolano Rodríguez. In: REUNIÓN IFLA DE EXPERTOS SOBRE UN CÓDIGO INTERNACIONAL DE CATALOGACIÓN, 3., 2005, Cairo, Egito. Disponível em: $<$ http://www.loc.gov/loc/ifla/imeicc/source/paris_principles_1961-sp.pdf >. Acesso em: 19 set. 2006.

DELSEY, T. Functional analysis of the MARC 21 bibliographic and holdings formats. $2^{\text {nd }}$. rev., 2003. Prepared for the Network Development and MARC Standards Office, Library of Congress. Disponível em: <www.loc.gov/marc/marc-functional-analysis/functionalanalysis.html $>$. Acesso em: 21 jul. 2008.

DENTON, W. FRBR and fundamental cataloguing rules. 2003. Disponível em: $<$ http://www.miskatonic.org/library/frbr.html> Acesso em: 02 de jul. 2005.

. FRBR and the history of cataloging. In: TAYLOR, Arlene (Org.). Understanding FRBR: what it is and how it will affect our retrieval tools. Westport, Ct: Greenwood Publishing Group, 2007. p. 35-57.

ESCOLANO RODRÍGUEZ, E. Edición preliminar de la ISBD consolidada. ENCUENTRO INTERNACIONAL DE CATALOGADORES, 3. Buenos Aires, Argentina, 2007. Disponível em: <www.bn.gov.ar>. Acesso em: 15 dez. 2007.

HENGEL, C., HENZE, G., PFEIFER, B. Comments on Functional Requirements for Authority Data (FRAD), 2. World-Wide Review. Disponível em: <http://www.dnb.de/standardisierung/pdf/comments_frad_dnb_1.pdf>. Acesso em: 20 jul. 2008.

INTERNATIONAL FEDERATION OF LIBRARY ASSOCIATIONS (IFLA) CATALOGUING SECTION. FRBR Review Group. Disponível em: $<$ www.ifla.org/VII/s13/wgfrbr/>. Acesso em: 06 jul. 2008.

FRBR Review Group. FRBR Bibliography. Disponível em: $\overline{<\mathrm{http} / / / w w w . i f l a . o r g / V I I / s 13 / w g f r b r / b i b l i o g r a p h y . p d f>. ~ A c e s s o ~ e m: ~} 27$ abr. 2008.

Meeting Report. Seoul, 2006. 6 p. Disponível em: <http://www.ifla.org/VII/s13/wgfrbr/FRBR_RG_Mtg2006.pdf $>$. Acesso em: 06 jul. 2008. 
Meeting Report. Durban, 2007. 5 p. Disponível em:

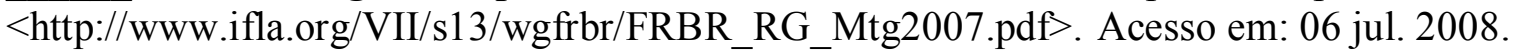

Minutes of the FRBR Review Group's Meeting. Oslo, 2005. 10 p. Disponível em:

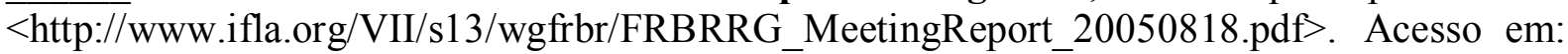
06 jul. 2008.

(2008a). Statement of International Cataloguing Principles. Disponível em: < http://www.ifla.org/VII/s13/icc/imeicc-statement_of_principles-2008.pdf>. Acesso em: 08 maio 2008.

. Working Group on Functional Requirements and Numbering of Authority Records (FRANAR). Functional Requirements for authority Data. Disponível em: $<$ http://www.ifla.org/VII/d4/FRANAR- ConceptualModel-2ndReview.pdf>. Acesso em: 16 jun. 2007.

. Working Group Functional Requirements for Subject Authority Records (FRSAR). Última atualização em 16 de janeiro de 2006. Disponível em: $<$ http://www.ifla.org/VII/s29/wgfrsar.htm>. Acesso em: 18 set. 2007.

IFLA STANDING COMMITTEE OF THE SECTION ON CATALOGUING. International Standard Bibliographic Description (ISBD): preliminary consolidated edition. München: IFLA, K.G. Saur, 2007. Disponível em: $<$ http://www.ifla.org/VII/s13/pubs/ISBD_consolidated_2007.pdf $>$.Acesso em: 10 dez. 2007.

. Terms of Reference for a Study of the Functional Requirements for Bibliographic Records, 04 sep. 1992.

IFLA STUDY GROUP ON THE FUNCTIONAL REQUIREMENTS FOR BIBLIOGRAPHIC RECORDS. Functional requirements for bibliographic records: final report. UBCIM Publications - New Series, vol. 19. München: K. G. Saur, 1998.

Functional requirements for bibliographic records: final report. Current text, feb. 2008. Including amendments and corrections to date. Disponível em: $<$ http://www.ifla.org/VII/s13/frbr/frbr_2008.pdf >. Acesso em: 10 mar. 2008.

JOINT STEERING COMMITTEE FOR DEVELOPMENT OF RDA. RDA document series. Última atualização em 29 de maio de 2008. Disponível em: $<$ http://www.collections canada.ca/jsc/rda.html\#background>. Acesso em: 23 ago. 2008. 
LE BCEUF, P. O Admirável Mundo Novo do FRBR: versão 5. In: REUNIÃo DA IFLA DE ESPECIALISTAS PARA UM CÓDIGO DE CATALOGAÇÃO INTERNACIONAL (IME ICC 5), 5., 2007, Pretória, África do Sul. Papers. Pretória, África do Sul: IFLA, 2007. 9 p. Traduzido por Fernanda Moreno. Disponível em: $<$ http://www.imeicc5.com/download/portuguese/Presentations2c_BraveNewFRBRWorld(PR _Port.pdf>. Acesso em: 06 mar. 2008.

LE COADIC, Y. Ciência da informação. Brasília: Briquet de Lemos, 1996.

LIMA, J.A. de O. Modelo Genérico de Relacionamentos na Organização da Informação Legislativa e Jurídica. 2008. Tese (Doutorado em Ciência da Informação) - Departamento de Ciência da Informação e Documentação, Universidade de Brasília, 2008.

MARCUM, D.B. Library of Congress response to On the Record: Report of The Library of Congress Working Group on the Future of Bibliographic Control. Washington, DC, 2008. 79 p. Disponível em: < http://www.loc.gov/bibliographic-future/news/LCWGResponse-MarcumFinal-061008.pdf >. Acesso em: 02 jul. 2008.

MCQAUIL, D.; WINDAHL, S. Communication models: for the study of mass communications. Londres: Longman, 1993.

MEY, E.S.A. Catalogação e descrição bibliográfica: contribuições a uma teoria. Brasília: Associação dos Bibliotecários do Distrito Federal, 1987. 201p. Originalmente apresentada como dissertação de Mestrado, Universidade de Brasília, 1986.

MORENO, F.P. Requisitos Funcionais para Registros Bibliográficos - FRBR: um estudo no catálogo da Rede Bibliodata. Dissertação (Mestrado em Ciência da Informação) Universidade de Brasília, 2006.

ONLINE COMPUTER LIBRARY CENTER (OCLC). FRBR Work-Set Algorithm. Disponível em: <http://www.oclc.org/research/projects/frbr/algorithm.htm>.Acesso em: 11 set. 2005.

PATTON, G. Extending FRBR to Authorities. Cataloging \& Classification Quarterly, v. 39, n. 3/4, p. 39-48, 2003.

SILVEIRA, N. C. Análise do impacto dos requisitos funcionais para registros bibliográficos (FRBR) nos pontos de acesso de responsabilidade pessoal. Dissertação. (Mestrado em Ciência da Informação) - Programa de Pós-Graduação em Ciência da Informação, PUC Campinas, 2007. 
SVENONIUS, E. The intellectual foundation of information organization. Cambridge, MA: MIT Press, 2000.

TILLETT, B. FRBR and RDA (Resource Description and Access. In: TAYLOR, A. (Org.). Understanding FRBR: what it is and how it will affect our retrieval tools. Westport, Ct: Greenwood Publishing Group, 2007. Cap. 7.

WORKING GROUP ON THE FUTURE OF BIBLIOGRAPHIC CONTROL. On the Record: Report of The Library of Congress Working Group on the Future of Bibliographic Control. Washington, DC, 2008. 49 p. Disponível em: <www.loc.gov/bibliographicfuture/news/lcwg-ontherecord-jan08-final.pdf $>$. Acesso em: 26 fev. 2008.

YEE, M. FRBR and Moving Image Materials. In: TAYLOR, A. (Org.). Understanding FRBR: what it is and how it will affect our retrieval tools. Westport, Ct: Greenwood Publishing Group, 2007. Cap. 11, p. 117-129.

ZHANG,Y; SALABA, A. Critical issues and challenges facing FRBR research and practice Bulletin of The American Society for Information Science and Technology, v. 33, n. 6, p. 30-31, ago/set. 2007.

ŽUMER, M. FRBR: the end of the road or a new beginning? Bulletin of The American Society for Information Science and Technology, v. 33, n. 6, p.27-29, ago/set. 2007.

\begin{abstract}
This paper briefly presents the Functional Requirements for Bibliographic Records - FRBR model. Then it describes their developments, that is, the models that resulted of the original one, some of the standards that were affected after its publication, bringing some actual discussions about these questions. Following, the user's tasks of FRBR model and another ideas found in literature are detailed, as well as tasks of FRAD model- Functional Requirements for Authority Data. Concluding, final commentaries about the FRBR model and the user's task are presented.
\end{abstract}

Key-words: Cataloguing. FRBR. FRAD. Catalogs. User tasks.

Originais recebidos em: 14/10/2008

Texto aprovado em: 08/05/2009 